\title{
Hormonas esteroideas foliculares y su relación con los resultados globales del ciclo de estimulación ovárica
}

\author{
Nayara López C. ${ }^{1}$, Onica Armijo S. ${ }^{1}$, Carolina González V. ${ }^{1 a}$, Rubén Gómez R. ${ }^{2}$, \\ Mariana Díaz-Almirón. ${ }^{b}$ \\ ${ }^{1}$ Servicio de Obstetricia, Ginecología y Reproducción, ${ }^{2}$ Servicio de Análisis Clínicos, ${ }^{b}$ Departamento de Bioestadística. \\ Hospital Universitario La Paz. Universidad Autónoma de Madrid. España.
}

a Bióloga. ${ }^{b}$ Matemática.

\section{RESUMEN}

Objetivo: Relacionar los niveles de hormonas esteroideas foliculares con el ciclo de estimulación ovárica y sus resultados globales. Métodos: Se incluyeron pacientes $\leq 38$ años, con esterilidad de causa masculina, tubárica o desconocida, que recibieron un protocolo largo con agonistas de $\mathrm{GnRH}$ y $\mathrm{rFSH}$. Se recogieron las muestras de la primera y segunda aspiración folicular de cada ovario y se realizó un quimioinmunoanálisis de estradiol, progesterona, testosterona y DHEAS. Resultados: Se obtuvieron cifras menores de DHEAS folicular en las pacientes con más días de frenado con agonistas de $\mathrm{GnRH}(p=0,0003)$. Cuantos más días de rFSH administrados, mayores fueron los niveles de testosterona y DHEAS folicular ( $p=0,03$; $p=0,03)$. En los resultados globales del ciclo, se obtuvo una correlación negativa entre las cifras de testosterona folicular y el número de complejos puncionados $(r=-0,360 ; p=0,002)$ y entre la testosterona folicular y el número de embriones de calidad $D(r=-0,233 ; p=0,047)$. El número de ovocitos maduros fue menor en pacientes con mayores niveles de testosterona folicular $(p=0,008)$. La progesterona folicular fue superior en ovocitos de buena calidad frente a los de calidad no destacable $(p=0,006)$ y muy mala calidad $(p=0,04)$. Conclusiones: Las cifras altas de testosterona folicular se correlacionaron con menor número de complejos puncionados, ovocitos maduros y embriones de calidad D. La buena calidad ovocitaria se asoció a niveles de progesterona folicular superiores.

\section{PALABRAS CLAVE: Estradiol, progesterona, andrógenos, líquido folicular}

\section{SUMMARY}

Objective: To relate the levels of follicular steroid hormones with the ovarian stimulation cycle and its overall results. Method: It was included patients $\leq 38$ years old with sterility of male, tubaric or unknown origin who underwent a long protocol with $\mathrm{GnRH}$ agonists and $\mathrm{rFSH}$. Samples were obtained from the first and second follicular aspiration of each ovary. A chemiluminescent immunoassay of estradiol, progesterone, testosterone and DHEAS was performed. Results: Figures of follicular DHEAS decreased as the days of treatment with $\mathrm{GnRH}$ agonists increased $(p=0.0003)$ and levels of follicular testosterone and DHEAS increased along with the days of treatment with $\mathrm{rFSH}(\mathrm{p}=0.03, p=0.03)$. In regard to the outcomes of the overall cycle it was found a negative correlation between follicular testosterone levels and the number of punctured complexes $(r=-0.360 ; p=0.002)$ and between follicular testosterone and the number of $D$ quality embryos $(r=-0.233 ; p=0.047)$. The number of mature oocytes was lower in patients with higher levels of follicular testosterone $(p=0.008)$. Follicular progesterone was higher in good quality oocytes as compared to those of no remarkable quality $(p=0.006)$ and very poor quality $(p=0.04)$. Conclusions: High levels of follicular testosterone were correlated with a fewer number of punctured complexes, mature oocytes and $D$ quality embryos. Good oocyte quality was associated with higher follicular progesterone levels.

KEY WORDS: Estradiol, progesterone, androgens, follicular fluid 


\section{INTRODUCCIÓN}

El resultado de cada ciclo de estimulación ovárica supone un reto en reproducción asistida, tanto para los profesionales dedicados a ello como para las parejas implicadas, no siendo predecible con facilidad.

Se han publicado varios estudios analizando los niveles de hormonas esteroideas foliculares y siguiendo al ovocito inmerso en ese líquido tras su fertilización mediante microinyección espermática (ICSI), pero poco se ha analizado sobre el resultado global del ciclo de estimulación de cada paciente (1-4). Un estudio ideal requeriría múltiples punciones vaginales individuales de cada uno de los folículos de la paciente para poder conocer fielmente el resultado específico al que se asocia cada folículo puncionado. Sin embargo, ello supondría un incremento de los riesgos asociados a la punción limitando de esta manera su aplicación en la práctica clínica (1). No se ha esclarecido por el momento si las diferencias en la composición hormonal folicular son una variable interpersonal o una variable entre los distintos folículos de la paciente (2).

El objetivo del estudio es valorar diversos parámetros del ciclo de estimulación ovárica y sus resultados globales y relacionarlos con los niveles hormonales foliculares de cada paciente.

\section{PACIENTES Y MÉTODO}

Se llevó a cabo un estudio prospectivo observacional en el que se incluyeron consecutivamente, desde febrero de 2011 a febrero de 2013 en el Hospital La Paz (Madrid), las pacientes que cumplieron los siguientes criterios de inclusión: $\leq 38$ años, índice de masa corporal $<30$ y esterilidad de causa masculina, tubárica o de origen desconocido. Las etiologías ováricas de cualquier índole fueron excluidas.

Se empleó un protocolo largo con agonistas de la hormona liberadora de gonadotropinas $(\mathrm{GnRH})$ y hormona folículo-estimulante recombinante (rFSH) de forma exclusiva. El crecimiento folicular se monitorizó por ecografía transvaginal. Con el hallazgo de $\geq 2$ folículos $>18 \mathrm{~mm}$ de diámetro y niveles de estradiol séricos consistentes, se administró gonadotropina coriónica humana (250 mcg, hCG, Ovitrelle; Merck-Serono S.A., Ginebra, Suiza); 36 horas después, se realizó la aspiración folicular eco-guiada de los complejos ovocito-corona-cumulus y del líquido folicular.

Para la punción folicular se utilizó una aguja fijada al transductor ecográfico por una guía, una bomba de vacío de regulación continua (modelo Labotect Aspirator 4014, Gottingen, Alemania) y un bloque térmico a $37^{\circ} \mathrm{C}$.

El primer y segundo folículo de cada ovario se aspiró individualmente. Los líquidos foliculares hemáticos fueron excluidos. La muestra fue centrifugada y trasladada a un tubo Falcon de $5 \mathrm{ml}$ y ubicada a $-70^{\circ} \mathrm{C}$ para posterior análisis.
El volumen de líquido aspirado fue correlacionado con el tamaño folicular como describió Wittmaack y cols (5).

Para la medida y cuantificación hormonal se empleó el inmunoanálisis de micropartículas quimioluminiscentes. El estradiol ( $\mathrm{ng} / \mathrm{ml})$, la progesterona $(\mu \mathrm{g} / \mathrm{ml})$ y la testosterona $(\mathrm{ng} / \mathrm{dl})$ se midieron en un equipo Architexct i2000 (Abbott Laboratories Philippines, Mandaluyong City), y la dehidroepiandrostendiona sulfato (DHEA-S) $(\mathrm{mg} / \mathrm{dl})$ en un equipo IMMULITE®2000 immunoassay system (SIEMENS, Erlangen, Germany). El coeficiente de variación intraensayo fue $<15 \%$. Se realizó una dilución al 1:1000 para el estradiol y la progesterona. La medición de testosterona y DHEAS no precisó dilución por su similitud con niveles séricos.

El método de fecundación elegido fue ICSI, llevada a cabo con ovocitos maduros (metafase II) valorados inmediatamente después de su recuperación y seleccionados por la presencia del primer corpúsculo polar. Los resultados de la fecundación se evaluaron 19-21 horas tras la ICSI y la fecundación se consideró normal por la presencia de dos pronúcleos y dos corpúsculos polares.

Los niveles de hormonas esteroideas foliculares se han relacionado con los días de administración de $\mathrm{GnRH}$ y rFSH, con las dosis de rFSH y con las cifras de estradiol el día de la administración de HCG.

Dentro de los resultados globales del ciclo, se evaluaron la calidad ovocitaria, el número de complejos puncionados, de ovocitos recuperados, maduros y degenerados y el número de embriones totales y de las distintas calidades embrionarias.

La calidad ovocitaria global se clasificó en cuatro grupos: no destacable, buena, mala y muy mala. Fue clasificada como buena ante la existencia de un citoplasma claro y homogéneo, granulosidad moderada e inexistencia de inclusiones. Se descendió en la clasificación a no destacable y mala calidad ovocitaria a medida que se perdieron dichas cualidades, clasificando como muy mala calidad ovocitaria al ovocito que presentó acumulaciones de retículo liso endoplasmático, granulosidad severa centralizada y vacuolización excesiva. Se clasificó la calidad ovocitaria de la forma habitual en la que suele hacerlo el biólogo en la práctica clínica; como clasificación global de los ovocitos obtenidos en el ciclo.

La calidad embrionaria se estableció según la clasificación de la Asociación Española para el Estudio de la Biología Reproductiva dividiendo los embriones en 4 calidades: A, B, C, D asignadas en Día+3 en función del número de células (6).

La correlación entre variables cuantitativas continuas se estudió mediante el coeficiente de correlación de Pearson. Se estimaron y compararon las medias de los niveles hormonales por punción con un modelo de ecuación de estimación generalizada, con matriz de trabajo intercambiable. Así, se ajustaron los datos de medidas repetidas. La significación 
estadística se estableció para valores de $\mathrm{p}<0,05$. Los datos fueron procesados mediante el programa SAS 9.2 (SAS Institute Inc., Cary,NC,USA).

El estudio recibió la aprobación del comité ético y las pacientes candidatas firmaron un consentimiento informado específico del estudio.

\section{RESULTADOS}

Fueron seleccionadas 34 pacientes excluyéndose 3 por necesidad de terapia adicional en el ciclo de estimulación ovárica. Las muestras hemáticas fueron también excluidas, por lo que finalmente se incluyeron 73 muestras foliculares procedentes de 31 pacientes.

La edad media de las pacientes fue de 33,1 $\pm 3,3$ años, los niveles de FSH sérica basales fueron de $6,6 \pm 1,8 \mathrm{UI} / \mathrm{L}$, con un recuento de folículos antrales medio de 12,2 $\pm 4,6$ folículos y una cifra media de hormona antimulleriana sérica de 2,8 $\pm 1,1 \mathrm{ng} / \mathrm{ml}$.

Se administró agonistas de $\mathrm{GnRH}$ durante una media de 21,23 \pm 5,78 días. La estimulación con rFSH se realizó durante una media de 9,90 $\pm 1,77$ días y empleando dosis media de 1633,26 $\pm 455,39$ UI. El número medio de folículos $>17 \mathrm{~mm}$ fue de
$8,55 \pm 3,94$ con un diámetro medio de 19,05 $\pm 1,03$ $\mathrm{mm}$ y un volumen medio de 5,02 $\pm 1,03$ cc.

De las 73 muestras foliculares, 59 contenían un ovocito maduro (81\%: 59/73) y 38 de ellos experimentaron una fecundación normal (64,4\%: $38 / 59)$. Se obtuvo un total de 31 embriones evolutivos en día +3 .

En relación a los resultados finales del ciclo por paciente, el número medio de complejos puncionados fue de $9,03 \pm 4,10$, con $8,90 \pm 4,18$ ovocitos totales. De ellos, 7,81 $\pm 3,66$ fueron ovocitos maduros, $y$ fecundaron normalmente $5,29 \pm 3,43$ obteniéndose $4,39 \pm 2,90$ embriones totales por paciente. Los ovocitos degenerados fueron de 0,26 $\pm 0,51$.

En cuanto a la calidad embrionaria media global, se obtuvo, embriones de calidad A: 1,13 $\pm 1,12$; embriones de calidad B: $0,94 \pm 1,18$; embriones calidad C: $0,90 \pm 1,01$ y embriones de calidad D: 1,39 $\pm 1,40$. Relación entre los niveles hormonales foliculares y las características del ciclo de estimulación (Tabla I): En los datos de la muestra se apreciaron cifras menores de DHEAS folicular en las pacientes con más días de frenado con agonistas de $\mathrm{GnRH}$ $(p=0,0003)$. Cuantos más días de rFSH administrados, mayores fueron los niveles de testosterona y DHEAS folicular $(p=0,03 ; p=0,03)$.

\section{Tabla I \\ RELACIÓN ENTRE NIVELES HORMONALES FOLICULARESY CARACTERÍSTICAS DEL CICLO DE ESTIMULACIÓN OVÁRICA}

Análisis de estimadores del parámetro ecuación de estimación generalizada

\begin{tabular}{|c|c|c|c|c|}
\hline Parámetro & Estimador & & & Valor $p$ \\
\hline Días de GnR & & & & \\
\hline Estradiol & 2278,90 & $-6105,18$ & 10662,99 & 0,59 \\
\hline Progesterona & 308,59 & $-472,53$ & 1089,72 & 0,43 \\
\hline Testosterona & 0,002 & $-0,091$ & 0,095 & 0,96 \\
\hline DHEAS & $-4,45$ & $-6,84$ & $-2,05$ & 0,0003 \\
\hline Dosis de rFS & & & & \\
\hline Estradiol & 33,86 & $-106,02$ & 173,74 & 0,63 \\
\hline Progesterona & $-4,03$ & $-15,42$ & 7,35 & 0,48 \\
\hline Testosterona & 0,0006 & $-0,0002$ & 0,001 & 0,15 \\
\hline DHEAS & 0,003 & $-0,02$ & 0,03 & 0,83 \\
\hline Días de rFSH & & & & \\
\hline Estradiol & $-22371,8$ & $-52403,3$ & 7659,61 & 0,14 \\
\hline Progesterona & $-334,95$ & $-2600,64$ & 1930,72 & 0,77 \\
\hline Testosterona & 0,37 & 0,01 & 0,52 & 0,03 \\
\hline DHEAS & 9,35 & 0,85 & 17,84 & 0,03 \\
\hline $\begin{array}{l}\text { Pico de estra } \\
\text { el día de HCG }\end{array}$ & & & & \\
\hline Estradiol & 6,18 & $-80,01$ & 92,38 & 0,88 \\
\hline Progesterona & 2,25 & $-3,31$ & 7,82 & 0,42 \\
\hline Testosterona & $-0,0007$ & $-0,001$ & $-0,0001$ & 0,02 \\
\hline DHEAS & $-0,03$ & $-0,05$ & $-0,009$ & 0,004 \\
\hline
\end{tabular}

GnRH: hormona liberadora de gonadotropinas. DHEAS: dehidroepiandrostendiona sulfato. rFSH: hormona folículoestimulante recombinante. HCG: gonadotropina coriónica humana 
En relación al pico de estradiol, el día de la administración de HCG, se dio una asociación negativa con las cifras de testosterona y DHEAS folicular $(p=0,02 ; p=0,004)$.

Relación entre los niveles hormonales en líquido folicular y los resultados globales del ciclo (Tabla II): Al evaluar los niveles hormonales foliculares y relacionarlos con el resultado global del ciclo, se obtuvo una correlación negativa estadísticamente significativa entre las cifras de testosterona y el número de complejos puncionados, y una correlación positiva entre el ratio estradiol/testosterona y dicho número de complejos ( $r=-0,360 ; p=0,002$ y $r=0,332$; $p=0,004$, respectivamente) (Figura $1 \mathrm{~A}, \mathrm{~B})$. Se estableció una correlación negativa, estadísticamente significativa entre la testosterona y el número de embriones de calidad $\mathrm{D}$, siendo la correlación con el ratio estradiol/testosterona positiva en relación a dicho número de embriones de calidad $D(r=-0,233$; $p=0,047$ y $r=0,264 ; p=0,024$ respectivamente) (Figura $1 \mathrm{C}, \mathrm{D})$.

Relación entre los valores hormonales en líquido folicular y la variable cantidad de ovocitos maduros (Tabla III): La asociación entre la cantidad de ovocitos maduros y los niveles hormonales de testosterona $(p=0,008)$ y el ratio estradiol/testosterona $(p=0,03)$ fue estadísticamente significativa. En el caso de la testosterona, se estableció como un estimador de carácter negativo, por lo que cuanto mayor fue el número de ovocitos maduros por paciente, menores fueron sus cifras de testosterona folicular. En cuanto al ratio estradiol/testosterona, fue un estimador de carácter positivo siendo mayor el ratio cuanto mayor fue la cantidad de ovocitos maduros.

Relación entre los niveles hormonales en líquido folicular y la calidad ovocitaria global del ciclo (Tabla IV): Al comparar los niveles hormonales foliculares por paciente con las calidades ovocitarias globales, se obtuvieron diferencias estadísticamente significativas en los niveles de progesterona entre calidad ovocitaria no destacable y buena calidad ovocitaria $(p=0,006)$ y entre buena y muy mala calidad ovocitaria $(p=0,04)$. En el ratio estradiol/ progesterona hubo diferencias estadísticamente significativas entre buena y mala calidad ovocitaria $(p=0,006)$.

Tabla II

NIVELES HORMONALES EN LÍQUIDO FOLICULAR Y RESULTADOS GLOBALES DEL CICLO

\begin{tabular}{|c|c|c|c|c|c|c|c|}
\hline & & Estradiol & Progesterona & Testosterona & DHEAS & $\mathrm{rE} / \mathrm{P}$ & $\mathrm{rE} / \mathrm{T}$ \\
\hline $\begin{array}{l}\text { Número de } \\
\text { complejos } \\
\text { puncionados }\end{array}$ & $\begin{array}{c}r \\
\text { valor } p\end{array}$ & $\begin{array}{l}-0,007 \\
0,955\end{array}$ & $\begin{array}{l}0,151 \\
0,201\end{array}$ & $\begin{array}{l}-0,360 \\
0,002^{*}\end{array}$ & $\begin{array}{c}-0,003 \\
0,979\end{array}$ & $\begin{array}{l}0,189 \\
0,109\end{array}$ & $\begin{array}{l}0,332 \\
0,004^{*}\end{array}$ \\
\hline $\begin{array}{l}\text { Número de } \\
\text { ovocitos } \\
\text { fecundados }\end{array}$ & $\begin{array}{c}r \\
\text { valor } p\end{array}$ & $\begin{array}{c}-0,084 \\
0,482\end{array}$ & $\begin{array}{l}-0,019 \\
0,871\end{array}$ & $\begin{array}{c}-0,185 \\
0,117\end{array}$ & $\begin{array}{l}0,037 \\
0,755\end{array}$ & $\begin{array}{l}0,084 \\
0,481\end{array}$ & $\begin{array}{l}0,136 \\
0,250\end{array}$ \\
\hline $\begin{array}{l}\text { Número de } \\
\text { ovocitos } \\
\text { degenerados }\end{array}$ & $\stackrel{r}{r}$ & $\begin{array}{c}-0,073 \\
0,537\end{array}$ & $\begin{array}{l}0,036 \\
0,764\end{array}$ & $\begin{array}{l}0,154 \\
0,194\end{array}$ & $\begin{array}{l}-0,229 \\
0,052\end{array}$ & $\begin{array}{l}-0,207 \\
0,079\end{array}$ & $\begin{array}{r}-0,141 \\
0,236\end{array}$ \\
\hline $\begin{array}{l}\text { Número de } \\
\text { embriones totales }\end{array}$ & $\stackrel{r}{r}$ valor $p$ & $\begin{array}{l}-0,079 \\
0,504\end{array}$ & $\begin{array}{l}-0,067 \\
0,576\end{array}$ & $\begin{array}{l}-0,146 \\
0,227\end{array}$ & $\begin{array}{l}0,116 \\
0,330\end{array}$ & $\begin{array}{l}0,046 \\
0,697\end{array}$ & $\begin{array}{l}0,057 \\
0,633\end{array}$ \\
\hline $\begin{array}{l}\text { Número } \\
\text { embriones totales } \\
\text { calidad A }\end{array}$ & $\stackrel{r}{r}$ valor $p$ & $\begin{array}{l}-0,054 \\
0,652\end{array}$ & $\begin{array}{l}-0,079 \\
0,505\end{array}$ & $\begin{array}{l}-0,073 \\
0,541\end{array}$ & $\begin{array}{l}0,141 \\
0,236\end{array}$ & $\begin{array}{l}0,151 \\
0,202\end{array}$ & $\begin{array}{l}0,044 \\
0,709\end{array}$ \\
\hline $\begin{array}{l}\text { Número } \\
\text { embriones totales } \\
\text { calidad B }\end{array}$ & $\begin{array}{c}r \\
\text { valor } p\end{array}$ & $\begin{array}{l}-0,122 \\
0,303\end{array}$ & $\begin{array}{l}-0,013 \\
0,910\end{array}$ & $\begin{array}{l}0,079 \\
0,508\end{array}$ & $\begin{array}{l}0,162 \\
0,171\end{array}$ & $\begin{array}{c}-0,222 \\
0,059\end{array}$ & $\begin{array}{c}-0,180 \\
0,128\end{array}$ \\
\hline $\begin{array}{l}\text { Número } \\
\text { embriones totales } \\
\text { calidad C }\end{array}$ & $\begin{array}{c}r \\
p \text { valor }\end{array}$ & $\begin{array}{c}-0,224 \\
0,056\end{array}$ & $\begin{array}{c}-0,120 \\
0,310\end{array}$ & $\begin{array}{c}-0,101 \\
0,394\end{array}$ & $\begin{array}{c}-0,124 \\
0,295\end{array}$ & $\begin{array}{c}-0,073 \\
0,541\end{array}$ & $\begin{array}{c}-0,078 \\
0,510\end{array}$ \\
\hline $\begin{array}{l}\text { Número } \\
\text { embriones totales } \\
\text { calidad D }\end{array}$ & $\begin{array}{c}r \\
\text { valor } p\end{array}$ & $\begin{array}{l}0,118 \\
0,319\end{array}$ & $\begin{array}{l}0,024 \\
0,842\end{array}$ & $\begin{array}{l}-0,233 \\
0,047^{\star}\end{array}$ & $\begin{array}{l}0,132 \\
0,266\end{array}$ & $\begin{array}{l}0,204 \\
0,084\end{array}$ & $\begin{array}{l}0,264 \\
0,024^{*}\end{array}$ \\
\hline
\end{tabular}


A:

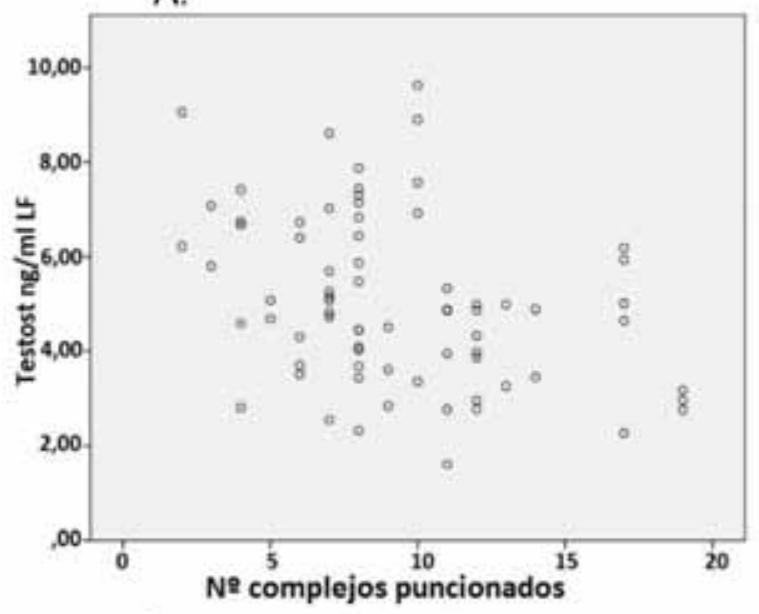

C:

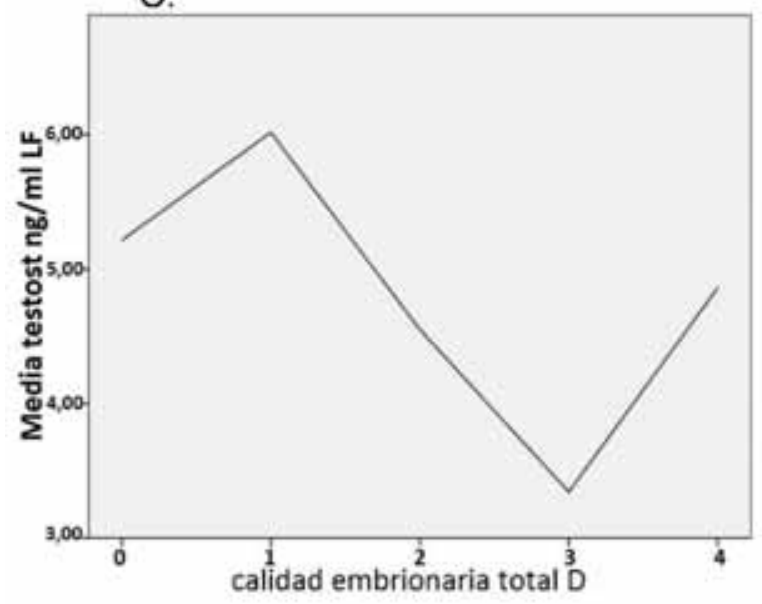

B:

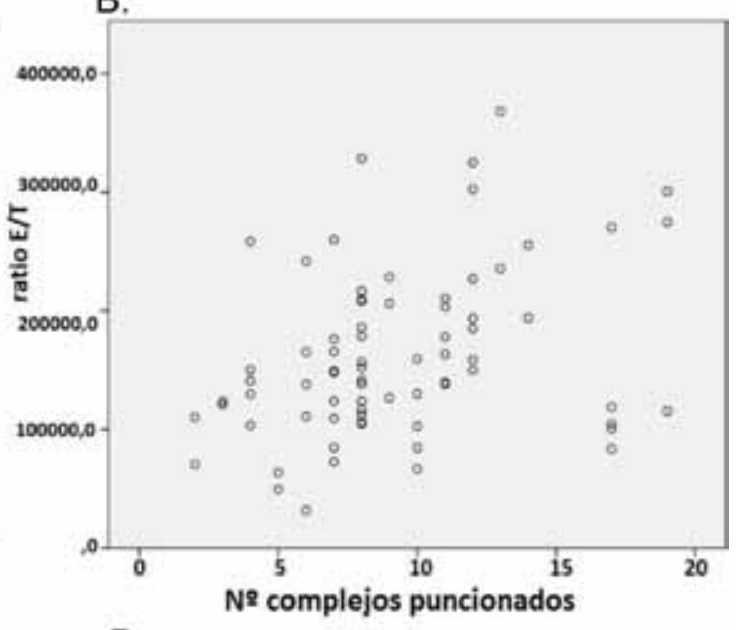

D:

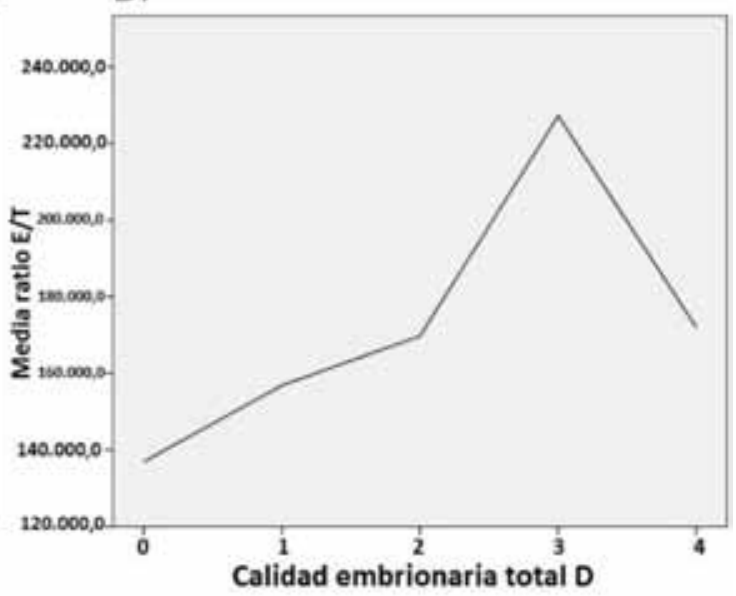

Figura 1. Relación entre los niveles hormonales en líquido folicular y los resultados globales del ciclo. A: Correlación negativa entre las cifras de testosterona y el número de complejos puncionados $(r=-0,360 ; p=$ $0,002)$. B: Correlación positiva entre el ratio estradiol/testosterona y número de complejos puncionados ( $r=$ $0,332 ; p=0,004)$. C: Correlación negativa entre la testosterona y el número de embriones de calidad $D(r=-$ $0,233 ; p=0,047)$. D: Correlación positiva entre el ratio estradiol/testosterona y el número de embriones de calidad $D(r=0,264 ; p=0,024)$. Testost: testosterona. $r E / T$ : ratio estradiol/testosterona.

\section{Tabla III \\ HORMONAS FOLICULARES Y NÚMERO DE OVOCITOS MADUROS}

Análisis de estimadores del parámetro ecuación de estimación generalizada

Cantidad de ovocitos maduros

\begin{tabular}{lcccc} 
Parámetro & Estimador & \multicolumn{2}{c}{ IC 95\% } & Valor p \\
\hline Estradiol $(\mathrm{pg} / \mathrm{ml})$ & $-131,999$ & -15210 & 14946,03 & 0,9863 \\
Progesterona $(\mathrm{ng} / \mathrm{ml})$ & 762,1955 & $-683,998$ & 2208,389 & 0,3016 \\
Testosterona $(\mathrm{ng} / \mathrm{ml})$ & $-0,1612$ & $-0,2818$ & $-0,0406$ & 0,0088 \\
DHEAS $(\mathrm{mcg} / \mathrm{dl})$ & $-0,4521$ & $-5,4360$ & 4,5318 & 0,8589 \\
Estradiol/progesterona & 0,8191 & $-0,6977$ & 2,3359 & 0,2899 \\
Estradiol/testosterona & 6054,854 & 348,4299 & 11761,28 & 0,0376 \\
\hline
\end{tabular}

DHEAS: dehidroepiandrostendiona sulfato 


\section{Tabla IV \\ NIVELES HORMONALES FOLICULARES Y CALIDAD OVOCITARIA GLOBAL POR PACIENTE}

\begin{tabular}{lcccc}
\hline $\begin{array}{l}\text { Niveles hormonales } \\
\text { en líquido folicular }\end{array}$ & $\begin{array}{c}\text { Calidad ovocitaria } \\
\text { No destacable }\end{array}$ & $\begin{array}{c}\text { Calidad ovocitaria } \\
\text { Buena }\end{array}$ & $\begin{array}{c}\text { Calidad ovocitaria } \\
\text { Mala }\end{array}$ & $\begin{array}{c}\text { Calidad ovocitaria } \\
\text { Muy mala }\end{array}$ \\
\hline Estradiol & $682.647,8$ & $776.411,2$ & $753.434,2$ & $714.320,2$ \\
(pg/ml) & $(489.996,4-$ & $(726.797,7-$ & $(701.156,3-$ & $(530.553,3-$ \\
& $875.299,3)$ & $826.024,7)$ & $805.712,0)$ & $898.087,1)$ \\
Progesterona & $20.362,48$ & $28.185,59$ & $22.892,90$ & $22.554,04$ \\
(ng/ml) & $(16.655,02-$ & $(24.007,35-$ & $(16.139,14-$ & $(19.136,31-$ \\
& $24.069,94)$ & $32363,82)$ & $29.646,66)$ & $25.971,77)$ \\
Testosterona & 5,28 & 5,99 & 4,45 & 5,02 \\
(ng/ml) & $(4,17-6,40)$ & $(4,41-7,58)$ & $(3,80-5,11)$ & $(4,39-5,66)$ \\
DHEAS & 157,24 & 129,72 & 111,72 & 146,09 \\
(mcg/dl) & $(115,01-199,47)$ & $(81,24-178,21)$ & $(91,30-132,15)$ & $(91,13-201,05)$ \\
Ratio Estradiol/ & 37,67 & 30,59 & 47,19 & 35,32 \\
Progesterona & $(21,52-53,83)$ & $(23,74-37,45)$ & $(37,32-57,06)$ & $(21,65-48,99)$ \\
Ratio Estradiol/ & $142.464,5$ & $141.603,5$ & $186.607,9$ & $155.056,2$ \\
Testosterona & $(86.403,17-$ & $(106.639,4-$ & $(157.642-$ & $(96.664,46-$ \\
& $198.525,9)$ & $176.567,6)$ & $215.573,7)$ & $213.447,9)$ \\
\hline
\end{tabular}

DHEAS: dehidroepiandrostendiona sulfato

\section{DISCUSIÓN}

Con el objeto de optimizar la evaluación de la relación entre los niveles hormonales foliculares y los distintos resultados reproductivos, se han intentado evitar posibles interferencias en los niveles hormonales. Para ello, se incluyeron pacientes con buena reserva ovárica cuantitativa como indican los parámetros medios de edad, FSH basal, recuento de folículos antrales y cifras de hormona antimulleriana sérica.

Las causas de esterilidad con componente ovárico fueron excluidas por su posible implicación en los niveles hormonales. Se empleó un protocolo largo con agonistas de $\mathrm{GnRH}$ y estimulación con rFSH de forma exclusiva, y se utilizaron pautas suaves de estimulación ovárica como muestran la dosis total de rFSH administrada, los días de estimulación y el número total de complejos puncionados.

Algunos trabajos han demostrado que las concentraciones de hormonas esteroideas foliculares son diferentes en enfermedades hormono-dependientes como la endometriosis o el síndrome de ovarios poliquísticos $(7,8)$. El empleo de un mismo protocolo en todas las pacientes y el uso exclusivo de rFSH permiten valorar con la menor interferencia posible el contenido hormonal folicular. En la actualidad están cobrado peso los protocolos de estimulación suave que buscan la mayor semejanza con el ciclo ovárico fisiológico intentando conseguir embriones de buena calidad, más que un alto número de embriones y permitiendo alcanzar cifras menores de estradiol durante la estimulación, lo que podría evitar alteraciones endometriales $(9,10)$.

La técnica de laboratorio empleada fue ICSI, realizada por la misma bióloga siguiendo el mismo procedimiento. Ello permitió investigar la relación entre los niveles hormonales foliculares y los resultados de fecundación específicos de cada ovocito, inmediatamente después de su recuperación. Gran parte de los estudios publicados han quedado limitados por la incertidumbre de si las muestras foliculares estudiadas eran originarias de folículos que contenían un ovocito maduro en el momento de la aspiración folicular porque la madurez ovocitaria no fue medida en el día de la recuperación ovocitaria $(11,12)$.

Se aspiró individualmente y se reservó para posterior análisis el líquido folicular procedente del primer y segundo folículo de cada ovario, excluyendo las muestras hemáticas. Tras la aspiración de cada folículo existe la posibilidad de que el ovocito correspondiente quede en el sistema y sea clasificado como perteneciente a otro folículo. Al analizar los resultados globales por paciente el efecto de cada ovocito queda reflejado en dichos resultados evitándose la pérdida de esa información. 
El análisis de estos líquidos foliculares se tomó como muestra de cada paciente y a partir de ellos se evaluaron las características globales del ciclo de estimulación. Un estudio ideal debería incluir el análisis de todos los líquidos foliculares de la paciente, pero su aplicación en la práctica clínica queda limitada por el aumento del riesgo asociado a múltiples punciones. En este contexto, la media de los niveles hormonales de varios folículos podría suponer una muestra adecuada por paciente.

Las cifras altas de testosterona folicular se correlacionaron con un menor número de complejos puncionados, de ovocitos maduros y de embriones de calidad D. En estos casos se dio una correlación positiva con el ratio estradiol/testosterona asumible muy probablemente por las bajas cifras de testosterona. Al observar los datos obtenidos, se aprecia una tendencia al alza en las cifras de testosterona, no sólo cuanto menor es el número de complejos puncionados sino también cuanto menor es el número de ovocitos fecundados y de embriones totales.

El número de ovocitos maduros guardó una correlación positiva con el ratio estradiol/testosterona, muy en esta línea, y posiblemente debido a las cifras de testosterona bajas. Estos resultados orientan a una implicación clara de la testosterona, no en los aspectos cuantitativos, sino en los cualitativos ovocitarios y embrionarios. Es decir, en los parámetros relativos al número, la testosterona se relacionó con un menor número, pero en cuanto a la calidad embrionaria, las pacientes con mayores cifras de testosterona folicular media tuvieron menos embriones de mala calidad.

En cuanto a las características del ciclo, sólo las cifras de DHEAS se vieron descendidas por los días de frenado con agonistas de $\mathrm{GnRH}$. Sin embargo, en cuanto a los días de administración de rFSH, a medida que aumentó el número de días, aumentaron las cifras de los dos andrógenos foliculares estudiados demostrándose la relación entre la FSH y los andrógenos (13). Por último, la cifra de estradiol sérico el día de la administración de HCG subió a medida que disminuyeron los andrógenos foliculares. El nivel de estradiol sérico depende del número de folículos maduros (200-300 $\mathrm{pg} / \mathrm{ml}$ por folículo con diámetro medio de $17 \mathrm{~mm}$ ), por lo que, una vez más, estos resultados están en la línea de la asociación de carácter negativo entre los parámetros cuantitativos y los andrógenos, especialmente la testosterona, en los resultados del estudio.

La calidad ovocitaria es la responsable en una proporción importante del desarrollo embrionario (14). Las cifras hormonales foliculares se compararon con la calidad ovocitaria global descrita por el biólogo al final del ciclo. Se obtuvo diferencias estadísticamente significativas en los niveles de progesterona entre ovocitos de buena y muy mala calidad y entre ovocitos de calidad buena y no destacable. Las cifras de progesterona fueron superiores en ovocitos de buena calidad. En la misma línea y muy probablemente a expensas de mayores cifras de progesterona en ovocitos de buena calidad, se obtuvo un ratio estradiol/progesterona menor en ovocitos de buena calidad con respecto a ovocitos de mala calidad.

Así, las pacientes con cifras altas de progesterona folicular presentaron mejor calidad ovocitaria. Las pacientes con buena calidad ovocitaria presentaron también cifras de testosterona folicular media elevadas, aunque las diferencias no alcanzaron la significación estadística. La asociación entre la progesterona folicular y la calidad ovocitaria ha sido descrita por otros autores (1), aunque no en términos de calidad ovocitaria global por paciente. Serían necesarios más estudios, con un mayor número de pacientes y folículos incluidos para esclarecer el papel de las hormonas esteroideas en los resultados globales del ciclo.

\section{CONCLUSIÓN}

Los niveles de testosterona folicular podrían postularse como marcadores negativos cuantitativos de complejos puncionados totales, de ovocitos maduros y de embriones de calidad D, y las cifras de progesterona folicular se podrían considerar como marcadores positivos de calidad ovocitaria.

\section{REFERENCIAS}

1. Lamb JD, Zamah AM, Shen S, McCulloch C, Cedars MI, Rosen MP. Follicular fluid steroid hormone levels are associated with fertilization outcome after intracytoplasmic sperm injection. Fertil Steril 2010;94:9527.

2. Mendoza C, Ruiz-Requena E, Ortega E, Cremades $\mathrm{N}$, Martinez F, Bernabeu R, et al. Follicular fluid markers of oocyte developmental potential. Hum Reprod 2002;17:1017-22.

3. Asimakopoulos B, Abu-Hassan D, Metzen E, Al-Hasani S, Diedrich K, Nikolettos N. The levels of steroid hormones and cytokines in individual follicles are not associated with the fertilization outcome after intracytoplasmic sperm injection. Fertil Steril 2008;90:604.

4. Wen X, Li D, Tozer AJ, Docherty SM, lles RK. Estradiol, progesterone, testosterone profiles in human follicular fluid and cultured granulosa cells from luteinized pre-ovulatory follicles. Reprod Biol Endocrinol 2010;8:117 doi: 10.1186/1477-7827-8-117.

5. Wittmaak FM, Kreger DO, Blasco L, Tureck RW, Mastroianni L Jr, Lessey BA. Effect of follicular size on oocyte retrieval, fertilization, cleavage and embryo quality in in-vitro fertilization cycles: a 6-year data collection. Fertil Steril 1994;62:1205-10.

6. Ardoy M, Calderon G, Cuadros J, Figueroa MJ, Herrer 
R, Moreno JM, et al. Cuadernos de Embriología Clínica. II Criterios ASEBIR de valoración morfológica de oocitos, embriones temprano y blastocistos humanos. 2nda ed. Edita: Asociación para el Estudio de la Biología de la Reproducción (ASEBIR). 2008. pp 9-16.

7. Naessen T, Kushnir MM, Chaika A, Nosenko J, Mogilevkina I, Rockwood AL, Carlstrom K, Bergquist J, Kirilovas D. Steroid profiles in ovarian follicular fluid in women with and without polycystic ovary syndrome, analyzed by liquid chromatography-tandem mass spectrometry. Fertil Steril 2010;94:2228-33.

8. Wunder DM, Mueller MD, Birkhäuser MH, Bersinger NA Steroids and protein markers in the follicular fluid as indicators of oocyte quality in patients with and without endometriosis. J Assist Reprod Genet 2005;22:257-64.

9. Palermo GD, Neri QV, Monahan D, Kocent J, Rosenwaks Z. Development and current applications of assisted fertilization. Fertil Steril 2012;97:248-59.

10. Verberg MF, Eijkemans MJ, Macklon NS, Heijnen EM, Baart EB, Hohmann FP, Fauser BC, Broekmans
FJ. The clinical significance of the retrieval of a low number of oocytes following mild ovarian stimulation for IVF: a meta-analysis. Hum Reprod Update 2009;15:5-12.

11. Andersen CY. Characteristics of human follicular fluid associated with successful conception after in vitro fertilization. J Clin Endocrinol Metab 1993;77:122734.

12. Rosen MP, Shen S, Dobson AT, Rinaudo PF, McCulloch CE, Cedars MI. A quantitative assessment of follicle size on oocyte developmental competence. Fertil Steril 2008;90:684-90.

13. Nielsen ME, Rasmussen IA, Kristensen SG, Christensen ST, Møllgård K, Wreford Andersen E, Byskov AG, Yding Andersen $\mathrm{C}$. In human granulose cells from small antral follicles, androgen receptor mRNA and androgen levels in follicular fluid correlate with FSH receptor mRNA. Mol Hum Reprod 2011;17:63-70.

14. Krisher RL, Bavister BD. Responses of oocytes and embryos to the culture environment. Theriogenology 1998;49:103-14 